\title{
Special issue for the twentieth edition of the workshop 'From objects to agents'
}

\author{
Matteo Baldoni ${ }^{\mathrm{a}}$, Federico Bergenti ${ }^{\mathrm{b}, *}$, Stefania Monica $^{\mathrm{b}}$ and Giuseppe Vizzari ${ }^{\mathrm{c}}$ \\ a Dipartimento di Informatica, Università degli Studi di Torino, 10149 Torino, Italy \\ ${ }^{\mathrm{b}}$ Dipartimento di Scienze Matematiche, Fisiche e Informatiche, Università degli Studi di Parma, \\ 43124 Parma, Italy \\ ${ }^{\mathrm{c}}$ Dipartimento di Informatica, Sistemistica e Comunicazione, Università degli Studi di Milano-Bicocca, \\ 20126 Milano, Italy
}

The first edition of the Workshop 'From Objects to Agents' (WOA) was held in Parma in May 2000. The workshop started as a joint initiative of the Agents and Multi-Agent Systems Working Group of the Italian Association for Artificial Intelligence (AIxIA) together with the Italian Association for Advanced Technologies based on Object-Oriented Concepts (TABOO). The workshop was intended to provide a forum for researchers and practitioners interested in understanding the possibilities that the intricate connection between agent technologies and objectoriented technologies can open. The first edition of the workshop counted more than fifty registered participants, from the academia and from the software industry, who attended the event for their interest in agent technologies and object-oriented technologies.

When TABOO ceased to exist, the workshop started to be primarily focused on all topics related to agents and multi-agent systems, and it initiated to be advertised as a stand-alone initiative with an international perspective organized by an independent community of researchers and practitioners based in Italy. As such, the twenty editions of the workshop have always been located in Italy, and the Steering Committee of the workshop have constantly

\footnotetext{
*Corresponding author: Federico Bergenti, Dipartimento di Scienze Matematiche, Fisiche e Informatiche, Università degli Studi di Parma, Parco Area delle Scienze 53/A, 43124 Parma, Italy. E-mail: federico.bergenti@unipr.it.
}

tried to involve all the major research groups that worked on agents and multi-agent systems in Italy. The workshop was hosted in the following venues (in alphabetical order): Camerino, Catania (three times), Genova, Milano (twice), Modena, Napoli, Palermo (twice), Parma (three times), Rende, Rimini, Scilla, Torino (twice), and Villasimius. In some occasions, the workshop was co-located with international events to promote the participation of researchers and practitioners from foreign countries.

The twentieth edition of the workshop, which was held on June $26^{\text {th }}-28^{\text {th }}, 2019$ in Parma ${ }^{1}$, can be considered as a significant milestone for the workshop and its community because it marks the maturity of the event. The twentieth edition took three full days, counted more than fifty participants, and it was structured in seven technical sessions, one keynote speech, two mini-school sessions, and two social events.

The seven technical sessions hosted the presentation of twenty-three papers that were collected in a virtual volume published by CEUR ${ }^{2}$. The topics discussed in the papers covered some of the most debated subjects in the research on agents and multi-agent systems, and they were not limited to the theme of the workshop, which was suggested in the call for papers as software agents in the age of artificial intelligence.

\footnotetext{
${ }^{1}$ www.ailab.unipr.it/woa19

${ }^{2}$ www.ceur-ws.org/Vol-2404
} 
The keynote speech was given by Giovanni Rimassa, who admittedly started his career at the first edition of the workshop, and it discussed the role of software agents in the industry, and the multidisciplinary agent-based approach that it underpins in the current world of international collaborative projects. The Steering Committee of the workshop decided to dedicate the keynote speech of the workshop, from the twentieth edition on, to the memory of Fabio Bellifemine, for his propulsive role in the community of software agents and for his consistent participation to the workshop and to its satellite activities. Giovanni Rimassa worked intensively with Fabio Bellifemine, and he was honored that he had the opportunity to give the first keynote speech dedicated to his memory.

The two mini-school sessions were intended to introduce students to selected topics of the research on agents and multi-agent systems, following a solid tradition of the workshop that dates back to 2004. In the first session, Silvia Rossi discussed the role of artificial intelligence for sensing and adaptation in human-robot interaction. In the second session, Francesco De Mola provided a perspective on Industry 4.0, and he discussed the role of artificial intelligence to support a smart factory.

The seven papers collected in this special issue are selected contributions that extend and improve some of the papers presented at the twentieth edition of the workshop. The papers of this special issue also include the outcomes of some of the discussions that followed the presentations at the workshop.

The first paper of this special issue extends [1], and it presents a survey on the integration of symbolic and sub-symbolic techniques for eXplainable Artificial Intelligence (XAI). The more intelligent systems based on sub-symbolic techniques pervade our everyday lives, the less human can understand them. This is the reason why symbolic approaches are getting more and more attention in the general effort to make artificial intelligence interpretable, explainable, and trustable. The understanding of the current state of the art of artificial intelligence techniques that integrate symbolic and sub-symbolic approaches is then of paramount importance. The paper provides an overview of the main symbolic/sub-symbolic integration techniques, focussing in particular on those that explicitly target XAI systems.

The second paper of the special issue extends [2], and it discusses the use of local trust measures to form agent Cloud of Things (CoT) groups. The devices that deal with complex tasks usually require power- ful hardware capabilities or, as a possible alternative, they require to get the resources that they need on the Cloud. When a device is virtualized on the Cloud, it can take benefit from relying on one or more software agents and on their social skills to mutually interact and cooperate. In particular, in a CoT scenario, where agents cooperate to perform complex tasks, the choice of a partner is a relevant problem. In such a context, when an agent is not capable to perform a reliable choice, then, like in real social communities, it can ask information to other agents that it considers as trustworthy. In order to support agents in their partner choices, the paper proposes a local trust model based on reliability and reputation measures coming from ego networks. The ego networks are used to partition the agents in groups by exploiting trust relationships to allow agents to be associated with reliable partners. To this aim, the paper presents an algorithm to form agent groups by exploiting available local trust measures. The presented results were obtained in a simulated scenario, and they confirm the advantages of the proposed approach.

The third paper of the special issue extends [3], and it discusses the role of trust and autonomy to regulate the users' acceptance of Internet of Things (IoT) technologies. The success of IoT technologies is undeniable. They are entering more and more in our lives, carrying out increasingly complex tasks. However, there are still a few problems that need to be solved. For instance, it is not given that the users will be prepared to afford all the automation that IoT devices will offer or that such a level of automation will be compatible with the users' cognitive attitudes and goals. The paper starts analyzing which reasons undermine the acceptance of IoT systems, and then it proposes a possible solution, taking into account not just user-device interaction but also device-device interaction. Since the complexity of the tasks that the user asks may require the cooperation of some devices to be realized, the regulation of this relationship represents a necessary step for this technology. The first contribution of the paper is the characterization of the autonomy a user can grant to an IoT device. The second contribution is a theoretical model to deal with users and to stimulate users' acceptance, taking also into account a possible, collaborative organizational structure, to manage the creation of groups and the partners' selection process.

The fourth paper of this special issue extends [4], and it discusses the use of interval arithmetic to support effective indoor positioning of software agents. The provision of advanced location-based services 
in indoor environments is based on the possibility of estimating the positions of mobile devices with sufficient accuracy and robustness. An algorithm to allow a software agent hosted on a mobile device to estimate the position of its device in a known indoor environment is proposed in the paper under the ordinary assumption that fixed beacons are installed in the environment at known locations. Rather than making use of geometric considerations to estimate the position of the device, the proposed algorithm first transforms the localization problem into a related optimization problem, which is then solved by means of interval arithmetic to provide the agent with accurate and robust position estimates. The adopted approach solves a major problem that severely limits the accuracy of the position estimates that ordinary geometric algorithms provide when the beacons are positioned to maximize line-of-sight coverage. Experimental results confirm that the proposed algorithm provides position estimates that are independent of the positions of the beacons, and they show that the algorithm outperforms a well-known geometric algorithm that was selected to validate results.

The fifth paper of the special issue extends [5], and it deals with the problem of eliciting cities points of interest from people movements and the related problem of suggesting effective itineraries. When a person visits an unknown city that has multiple interesting locations, it is not so easy to find a location that is lively and convenient to visit in a given time-frame. To overcome such a problem, the paper proposes to make use of two technologies: smartphones, equipped with sensors to read GPS coordinates, and multi-agent systems, to provide assistance to users and to gather collective knowledge. Data collected by means of devices are analyzed and organized in such a way to find locations that could be of immediate interest to people. Agents gather opinions from several users in terms of scores quantifying the level of satisfaction on visiting some place on a given time-frame. Suggestions are made to potentially interested users by selecting for them locations according to closeness and satisfaction scores. In this approach, interesting locations emerge from the analysis of gathered data, hence scores and suggestions can be available for any city, provided that enough people feed data to the system. Such interesting locations are found dynamically according to the behavior of people.

The sixth paper of the special issue extends [6], and it proposes a reputation-based framework to support dynamic car-pooling. In the last decades, individual urban traffic flows have increased all over the world with a consequent growth of road congestion and environmental pollution. In this context, car-pooling is an interesting car-based alternative to satisfy the individual mobility demand by optimizing the car loading factor with respect to the number of passengers, provided that all the participants share trip origin and destination in the same time slot. To make the approach more appealing, the paper proposes an on-demand car-pooling service that offers variable fares on the basis of trip length and number of participants. Multi-agent, reputation and recommender system technologies in synergy with a routing algorithm were used to this aim. Discussed experiments on simulated data provided convincing evidence of the potential advantages of the proposed approach.

The seventh paper of the special issue extends [7], and it discusses an actor-based solution for node embedding over large networks. The application of machine learning techniques over networks, such as prediction tasks over nodes and edges, is becoming crucial in the analysis of complex systems in a wide range of research fields. One of the enabling technology in such scenarios is represented by node embedding, which enables to automatically learn features over a network. Among the different approaches proposed in literature, the most promising are DeepWalk and Node2Vec, where the embedding is computed by combining random walks and neural language models. However, common limitations with such techniques are related to memory requirements and time complexity. The paper proposes a distributed and scalable solution, named ActorNode2 Vec, that keeps all the advantages of Node2 Vec but uses the actor model to distribute the computational load. The paper shows the efficacy of the proposed approach with a real network by making a comparison with Deep walk and an Apache Spark distributed implementation of Node2 Vec. Discussed results show that with ActorNode2 Vec computational times are drastically reduced without losing embedding quality.

In conclusion, the Guest Editors of this special issue would like to gratefully thank all authors and reviewers-those who helped with the twentieth edition of workshop, and those involved in the production of this volume-and, more generally, they would like to thank the lively, creative and sometimes volcanic community that has been regularly meeting for twenty years at the workshop. Finally, the Guest Editors would also like to invite other researchers and practitioners to consider the workshop for presenting their most disruptive ideas and relevant results for the twenty years to come, or for even more. 


\section{References}

[1] R. Calegari, G. Ciatto, J. Dellaluce and A. Omicini, Interpretable Narrative Explanation for ML Predictors with LP: A Case Study for XAI, in: Proceedings of the 20th Workshop From Objects to Agents (WOA 2019), CEUR Workshop Proceedings, Vol. 2404, RWTH Aachen, (2019), pp. 105-112.

[2] G. Fortino, L. Fotia, F. Messina, D. Rosaci and G.M.L. Sarné, Supporting Agent CoT Groups Formation by Trust, in: Proceedings of the 20th Workshop From Objects to Agents (WOA 2019), CEUR Workshop Proceedings, Vol. 2404, RWTH Aachen, (2019), pp. 71-76.

[3] A. Sapienza and R. Falcone, A Theoretical Model for the Human-IoT Systems Interaction, in: Proceedings of the 20th Workshop "From Objects to Agents"(WOA 2019), CEUR Workshop Proceedings, Vol. 2404, RWTH Aachen, (2019), pp. 90-97.

[4] S. Monica and F. Bergenti, Experiments on Robust Indoor Localization of Mobile Devices Using Interval Arithmetic, in: Proceedings of the 20th Workshop From Objects to Agents (WOA 2019), CEUR Workshop Proceedings, Vol. 2404, RWTH Aachen, (2019), pp. 14-21.

[5] C. Cavallaro, G. Verga, E. Tramontana and O. Muscato, Multi-Agent Architecture for Point of Interest Detection and Recommendation, in: Proceedings of the 20th Workshop From Objects to Agents (WOA 2019), CEUR Workshop Proceedings, Vol. 2404, RWTH Aachen, (2019), pp. 98-104.

[6] M.N. Postorino and G.M.L. Sarné, A Preliminary Study for an Agent Blockchain-Based Framework Supporting Dynamic Car-Pooling, in: Proceedings of the 20th Workshop From Objects to Agents (WOA 2019), CEUR Workshop Proceedings, Vol. 2404, RWTH Aachen, (2019), pp. 65-70.

[7] G. Lombardo and A. Poggi, A Scalable and Distributed Actor-Based Version of the Node2Vec Algorithm, in: Proceedings of the 20th Workshop From Objects to Agents (WOA 2019), CEUR Workshop Proceedings, Vol. 2404, RWTH Aachen, (2019), pp. 134-141. 